\title{
Exergy Analysis of Thermo-Fluid Energy Conversion Systems in Model-Based Design Environment
}

\author{
Daniel Bender \\ Institute of System Dynamics and Control, DLR German Aerospace Center, Germany Daniel. Bender@dlr. de
}

\begin{abstract}
Exergy-based analysis has been emerging as a powerful tool for the evaluation of energy intensive systems. Exergy is the maximum theoretical useful work obtainable as the system is brought into complete thermodynamic equilibrium with the thermodynamic environment. Besides the thermodynamic efficiency, both the real thermodynamic value of an energy carrier and the real thermodynamic inefficiencies within a system can be identified. Environmental control systems (ECS) of aircraft as highly interacting systems are an ideal candidate for exergy-based analysis. The design task on architectural level is currently performed using model-based design methods. However, if such systems are evaluated from an exergetic point of view, the analysis is done subsequent of the model-based simulations using rudimentary tools. This work presents a way how exergy-based methods can be integrated into the model-based design environment of Modelica with focus on generic compatibility.

Keywords: exergy analysis, thermo-fluid systems, energy conversion systems, aircraft ECS
\end{abstract}

\section{Introduction}

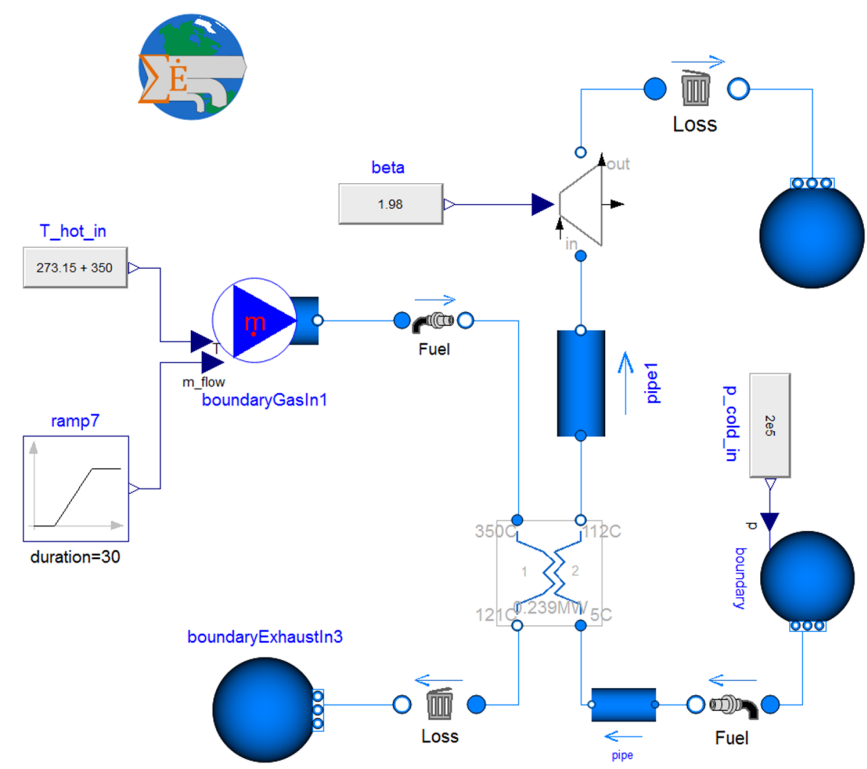

Figure 1. Modelica diagram of power generation cycle partly modeled with components from MSL.
Exergy analysis is a very specific field of evaluation methods for energy conversion processes and usually not well known by the broad audience. A simple example of its evaluation capability is given right here in the beginning to support the understanding of the further work.

Figure 1 shows the Modelica diagram of a simple power generation process. Cold air is preheated within a heat exchanger and then expanded while passing a turbine to produce power. Using exergy-based methods one can give information about the real thermodynamic value of the energy supplied (Fuel, E_fuel) to the system, how much is discharged to the environment (Loss, E_loss) and wasted due to inefficiencies, the so-called exergy destruction (E_D).

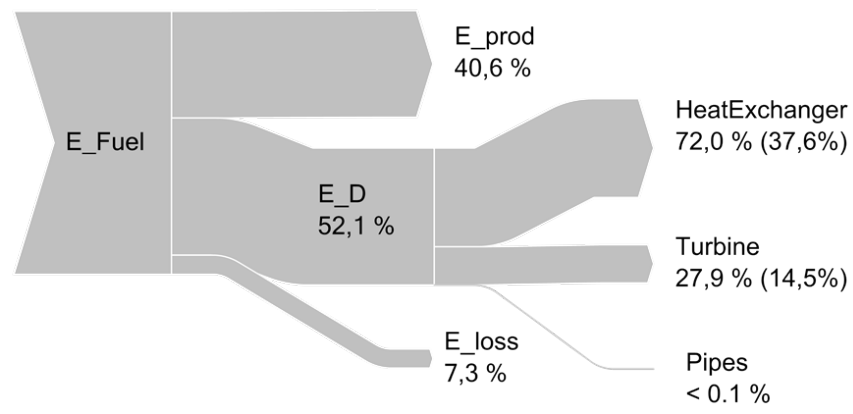

Figure 2. Grassmann diagram showing the results of the exergy analysis for the power generation cycle of Figure 1.

Figure 2 shows a Grassmann diagram (Grassmann, 1950) of the simulation results for the power generation cycle. The analysis shows that $40.6 \%$ of the supplied fuel exergy is only used to produce power in the turbine. $52.1 \%$ is wasted due to inefficiencies in the components and $7.3 \%$ is discharged by the exiting flows leaving the system. Further the results identify the heat exchanger as the main source of inefficiencies. It is responsible for $72.0 \%$ of the exergy destruction, which is $37.6 \%$ of the supplied fuel exergy. The turbine causes less with about $27.9 \%$ and $14.5 \%$, respectively. The losses within the pipes can be neglected. The outcomes of the exergy analysis give not only information about the total inefficiency of the process, but also about the impact of the single components.

This example shall convey an idea of exergy-based methods and their significance. The focus of this work is to present the integration of exergy-based methods into the 
model-based design environment of Modelica. Within this section, state of the art tools for the modeling of thermofluid systems and evaluation of such are discussed. Then, a brief introduction to the methodology of exergy analysis is given and their application within this work. The next section presents the integration of the exergy-based methods into the model-based environment and two examples are shown. Finally, the paper is concluded with some remarks.

Model-based design methods have become well established methods for numerical simulations of complex thermo-fluid systems. This comprises the simulation of single components and large scale systems with large time scales. Much effort have been put into the modeling of energy intensive systems for different fields of applications. Ranging from basic thermo-fluid modeling (Elmqvist et al., 2003) to automotive refrigeration systems (Limperich et al., 2005), large buildings simulation (Wetter et al., 2014) and environmental control systems of aircraft (Sielemann et al., 2007).

The modeling and simulation process as part of a design process usually comes along with subsequent evaluation and optimization tasks of the system of interest. Optimization capabilities for different kinds of applications are provided by specific libraries (Pfeiffer, 2012; Bender, 2016). The evaluation of energy conversion systems is performed in most cases using evaluation criteria based on the first law of thermodynamics. Energy balances are formulated and the efficiency of a conversion process is measured by comparing the supplied energy with the desired output of the process. The difference of the supplied energy and output represents the waste energy, in other words the losses of the process. Most component models of energy conversion processes, such as turbo components or heat exchangers are either described by thermodynamic efficiencies based on the first law or are equipped with such. Hence, an additional library or tool is not necessary. Unfortunately, this does not apply for analysis that asks for extended questions such as the following topics. Environmental or economical issues are usually not mandatory for the simulation of the energy conversion process and are influenced by additional factors such as costs and system specific aspects linked to their field of application. (Wischhusen et al., 2003) present the simulation and optimization of a complex industrial energy system with respect to economic benefits. The analysis was performed using an applied simulation tool based on Modelica. A physical domain independent library was developed by (Zimmer and Schlabe, 2012). Economic models are provided for the implementation into Modelica and allow energy management tasks.

Exergy analysis can be seen as an extended thermodynamic analysis that requires a different view of the energy conversion process. The exergy equations need only basic math and are usually performed subsequent of the simulation process. When it comes to exergy analysis of dynamic systems, a subsequent analysis needs more effort as the dataset increases significantly. (Sanghi et al., 2014) developed a Modelica-based tool to provide a dynamic exergy analysis for buildings simulation. The library followed a similar approach as it is presented in this paper. Unfortunately, the library is not published and the formulation of the exergy equations are not documented. Therefore, no statement is possible if it meets the requirements for the application to aircraft environmental control systems. This circumstance leads directly to the motivation of the work presented here.

The question of how exergy analysis can be integrated into the model-based design environment developed during the endeavors for a comprehensive formulation of exergy-based methods for aircraft environmental control systems.

The definition of exergy balances highly depends on the actual thermodynamic states of the working fluid and the conditions of the reference state. Aircraft operate among highly varying environmental conditions that impact temperature, pressure and humidity. The same applies to aircraft ECS. With changing ambient environment, the ECS operates on different operation points. Depending on the definition of the reference environment for the exergy analysis, any possible situation must be considered for the integration of exergy-based methods. The impact of the reference environment on the definition of the exergy balances is consolidated later in this paper.

Based on the demands derived from the exergy-based methods and the ongoing development of aircraft ECS using model-based design, a library is presented with the aim to allow exergy-based analysis of aircraft environmental control systems. The application of this library shall not be limited to aircraft applications and library structures currently used for the modeling and simulation of aircraft ECS (Sielemann et al., 2007; Zimmer et al., 2018). Both libraries have been developed in collaboration with an industry partner and are not publicly available at their current development stage.

\section{Exergy analysis}

Energy balances consider the quantity of energy, but neglects to express the quality of energy. The real thermodynamic value, i.e. its quality, of an energy source gives information about its potential to cause a change in a useful way. Kinetic, potential, mechanical and electric energy can be transformed in an ideal process to any other form of energy. The quality of thermal and chemical energy, however, depends on the state of the energy carrier (temperature, pressure and chemical composition) with respect to the environment. In thermodynamics, exergy characterizes the quality of a given quantity of energy. Using an energy balance in combination with the second law of thermodynamics, both the thermodynamic true value of an energy carrier, and the real thermodynamic inefficiencies can be determined. This is possible for a single process and on system level. Within the system boundaries, the occurring 
Table 1. Definition of exergy of fuel and exergy of product.

Component schematic

real thermodynamic inefficiencies are exergy destruction and exergy transfers out of the system are regarded as exergy losses (Bejan et al., 1996).

(Tsatsaronis, 2007) specified the definition that the exergy of a thermodynamic system is the maximum theoretical useful work obtainable as the system is brought into complete thermodynamic equilibrium with the thermodynamic environment while the system interacts with this environment only.

The total flow exergy of a fluid stream $i$ is expressed as:

$$
\dot{E}_{t, i}=\dot{m} \cdot\left[h_{i}-h_{0}-T_{0} \cdot\left(s_{i}-s_{0}\right)\right]
$$

where $\dot{m}_{i}$ is the mass flow, and $h$ and $s$ represent the specific enthalpy and specific entropy of the fluid stream $i$ and reference environment 0 .

The exergy flow balance for the $k-t h$ component is defined by:

$$
\dot{E}_{\mathrm{F}, k}=\dot{E}_{\mathrm{P}, k}+\dot{E}_{\mathrm{D}, k}
$$

where subscripts F, P and D represent the fuel exergy, product exergy and destroyed exergy of the $k-t h$ component. (Lazzaretto and Tsatsaronis, 2006)

The balance for the total system can be written as:

$$
\dot{E}_{\mathrm{F}, t o t}=\dot{E}_{P, t o t}+\sum_{k} \dot{E}_{\mathrm{D}, k}+\dot{E}_{\mathrm{L}, t o t}
$$

with tot representing the total amount of the overall system.

The exergetic efficiency $\varepsilon_{k}$ of the $k-t h$ component is defined by the following expression:

$$
\varepsilon_{k}=\frac{\dot{E}_{\mathrm{P}, k}}{\dot{E}_{\mathrm{F}, k}}=1-\frac{\dot{E}_{\mathrm{D}, k}}{E_{\mathrm{F}, k}}
$$

The rate of exergy destroyed related to the exergy of total fuel is expressed by the exergy destruction ratio:

$$
y_{\mathrm{D}, k}=\frac{\dot{E}_{\mathrm{D}, k}}{\dot{E}_{\mathrm{F}, t o t}}
$$

The approach of defining the exergy balance using fuel and product exergy instead of entering and exiting energy flows is explained in detail by (Lazzaretto and Tsatsaronis, 2006) and (Tsatsaronis and Morosuk, 2013). The product exergy $\dot{E}_{P}$ represents the desired result (expressed in terms of exergy) generated by the system being considered. The fuel exergy $\dot{E}_{F}$ represents the general resources in terms of exergy that are expended to provide the product exergy. The fuel and product parts of a component are determined by considering the physical exergy $\dot{E}^{\mathrm{Ph}}$, i.e. thermal $\dot{E}^{T}$ and mechanical $\dot{E}^{M}$ parts, and chemical exergy $\dot{E}^{\mathrm{Ch}}$ of each stream. The definition of the balances is done for each component differently depending on its aim, i.e. the balances for a heat exchanger differ from the balances for an expansion device. Additionally 
to the component's aim, the reference environment has to be taken into account. (Bender, 2017) gives a comprehensive overview for typical energy conversion processes of an aircraft environmental control system. Table 1 shows

Table 2. Component categories and number of operation conditions considered for the definition of exergy rates.

\begin{tabular}{ll}
\hline Component categories & \# of Cases \\
\hline Heat exchanger & 6 \\
Compressor & 3 \\
Turbine & 3 \\
Water extraction & 1 \\
Water injection & 3 \\
Mixer & 1 \\
Flow resistance & 1 \\
\hline
\end{tabular}

exemplary the definition of the exergy rates for a turbo driven compressor or fan. During the compression, external power is supplied to the process and increases the pressure and concurrently the temperature of the entering fluid stream. Applying now the methodology of fuel and product exergy, the supplied power belongs to the fuel and the pressure increase counts as product exergy. The chemical exergy does not change among the process for most cases. It needs to be considered for a comprehensive approach because it could change in the case of having a saturated moist air flow and evaporation takes place during the compression. The details of the behavior of the chemical exergy are not discussed here as they would exceed the scope of this work. However, the thermal exergy that depends on the temperature changes differently from the pressure. It reaches its minimum of 0 at the reference temperature and increases with increasing deviation from $T_{0}$. Depending on the operation condition, three exergy balances can be identified. The operation conditions define in this context at which temperature the compressor operates with respect to the reference environment, in particular the reference temperature $T_{0}$. The first case considers the operation above the reference environment. The second case includes the crossing of $T_{0}$. During the third case, the temperature at the outlet remains below $T_{0}$.

\section{Integration into model-based envi- ronment}

The analysis of thermo-fluid energy conversion systems using exergy-based methods is usually performed in two steps. The system is simulated in a first step using a simulation environment, such as Ansys (Ansys, 2018), Aspen Plus (aspentech, 2018) or Modelica. The produced thermodynamic data is then used to do the exergy analysis in a subsequent step. This requires a data transfer to another calculation software (e.g. EES (F-Chart, 2018) or MS Excel). If the analysis is limited to one or a few operation points, the amount of data remains manageable. But if the exergy analysis shall be performed for several operation points or include dynamic behavior of a system, the produced data exceed soon a practicable amount.

Modelica is already used for the application of large thermo-fluid systems. Evaluation criteria based on the first law of thermodynamics such as energetic efficiencies are provided in most components that describe energy conversion processes. The advantage of the model-based approach that system models can be built from scratch or modified using available libraries within a short time shall now be extended with the capability of exergetic-based methods. The exergy analysis is a subsequent calculations step and does not impact the system behavior.

\subsection{Requirements}

In order to achieve a solution as generic as possible some requirements need to be formulated derived from both, the exergy-based methods and the model-based environment:

\section{Exergy-based Methods}

- Retrieve thermodynamic state of all energy streams entering and exiting a component

- Identify the aim the component's energy conversion process

- Select the appropriate exergy balance of fuel and product exergy rates depending of the operation condition and reference environment

- Allow a user defined exergy analysis on system level using the component's based analysis

- Centralized propagation of reference environment on system level among all components

- Media models must provide appropriate functions to calculate further thermodynamic data

\section{Model-based environment}

- Generic approach for easy integration into any thermo-fluid library

- Compliant with Modelica Standard Library (Usage of MSL Media models and MSL Fluid connectors)

- Minor impact on numerical computation

The best way to put these requirements into action would require the screening of the modeled system architecture. The structure could be gathered to identify the components' aims and link them with their appropriate fuel and product definition. This procedure can be seen as a preceding step before the simulation starts. Unfortunately, Modelica at its recent stage of development used for this work (The Modelica Association, 2013) does not provide sufficient practical sequential capabilities. Therefore the integration needs to be realized in a different way. 
Listing 1. Modelica code of exergy implementation in sensor models: Calculation of exergy flows.

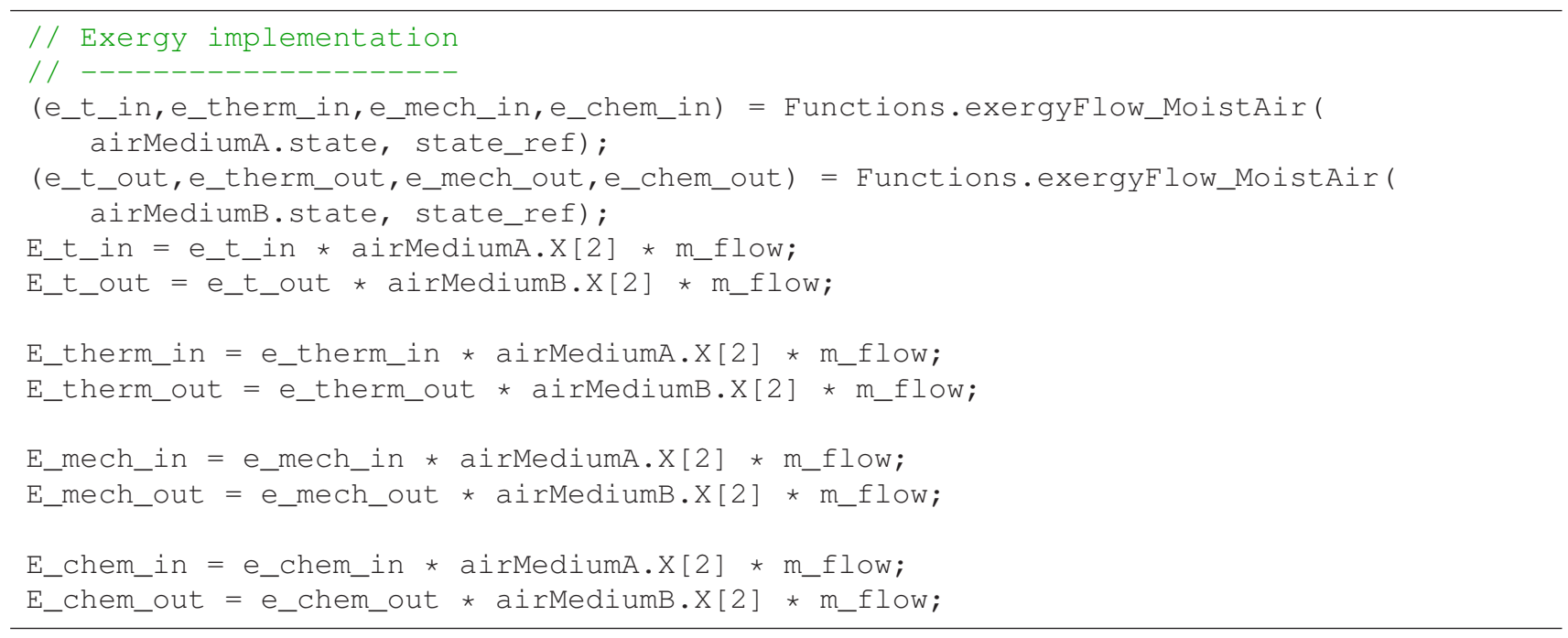

\subsection{Component level}

The exergetic analysis on component level includes the exergy balance of Equation (2) and the calculation of the exergetic efficiency with Equation (4). Table 1 gives an example of how the fuel and product exergy is defined for a compressor. To calculate the appropriate exergy flows, the thermodynamic data at the component's connectors must be provided. As mentioned before, it is hardly manageable to catch the data from outside the component without huge effort for the user. Therefore the approach of a sensor model was chosen to integrate the exergy equations to the component. To keep the usability as simple as possible, the sensor model must be dropped to the component model and linked to the component's connectors and some other variables. Figure 3 shows how the staticPipe model looks after the exergy sensor has been integrated on component level. The sensor model is then linked with the component by adding standardized code to the component model to supply the reference environment and the WorldEx model. The following listing shows an example of an exergy sensor integrated to a compressor model:

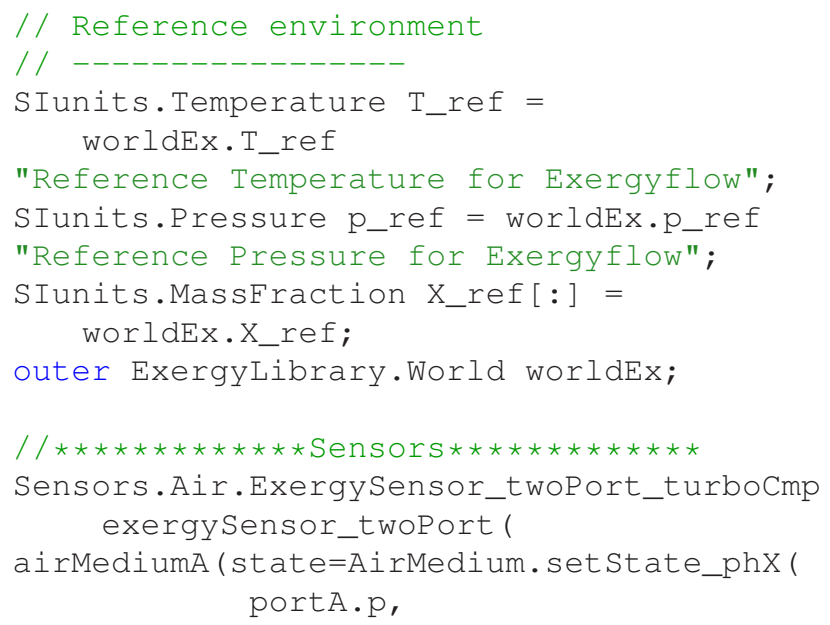

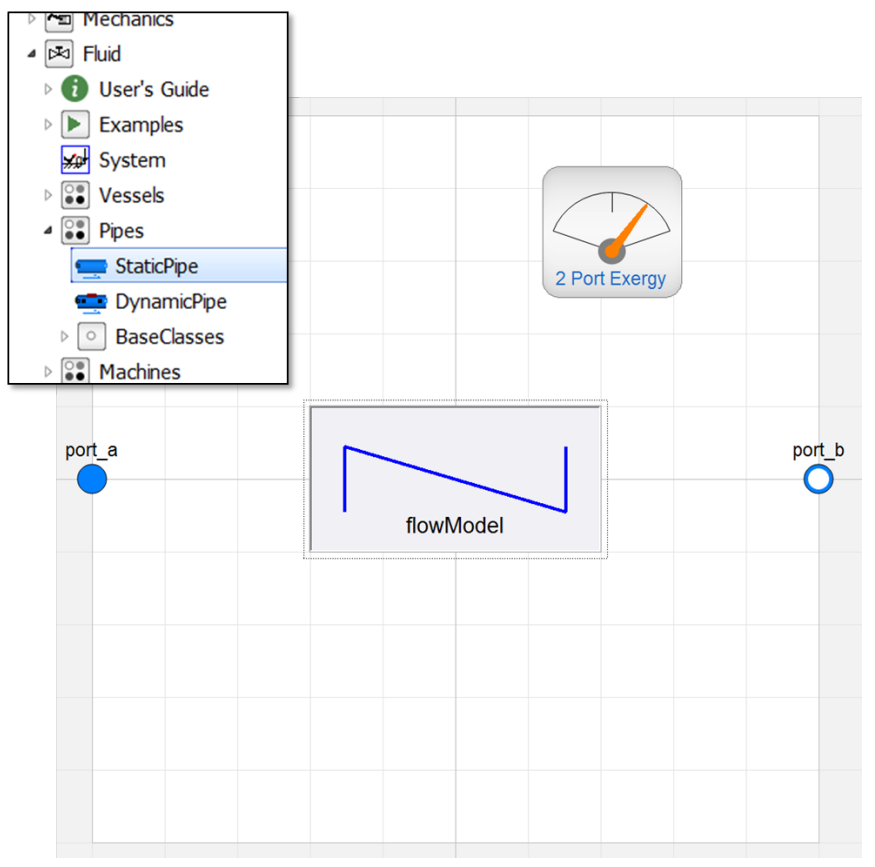

Figure 3. Modelica diagram of StaticPipe model with exergy sensor integrated.

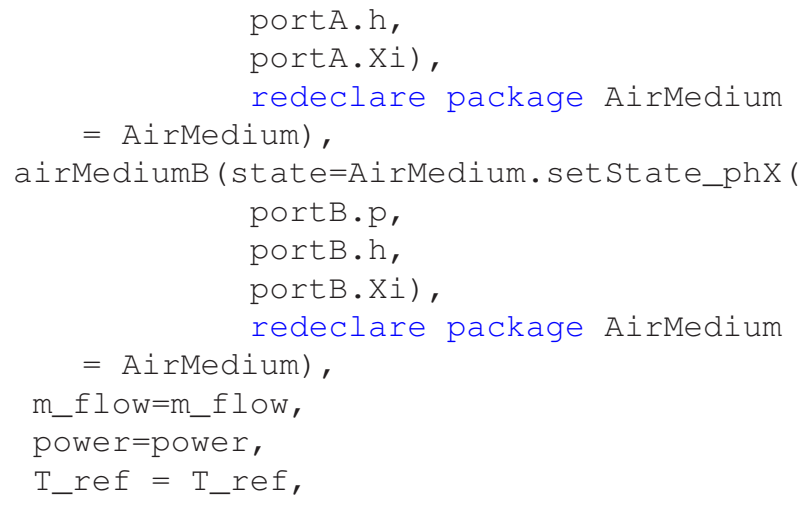


Listing 2. Modelica code of exergy implementation in sensor models: Definition of fuel and product balances.

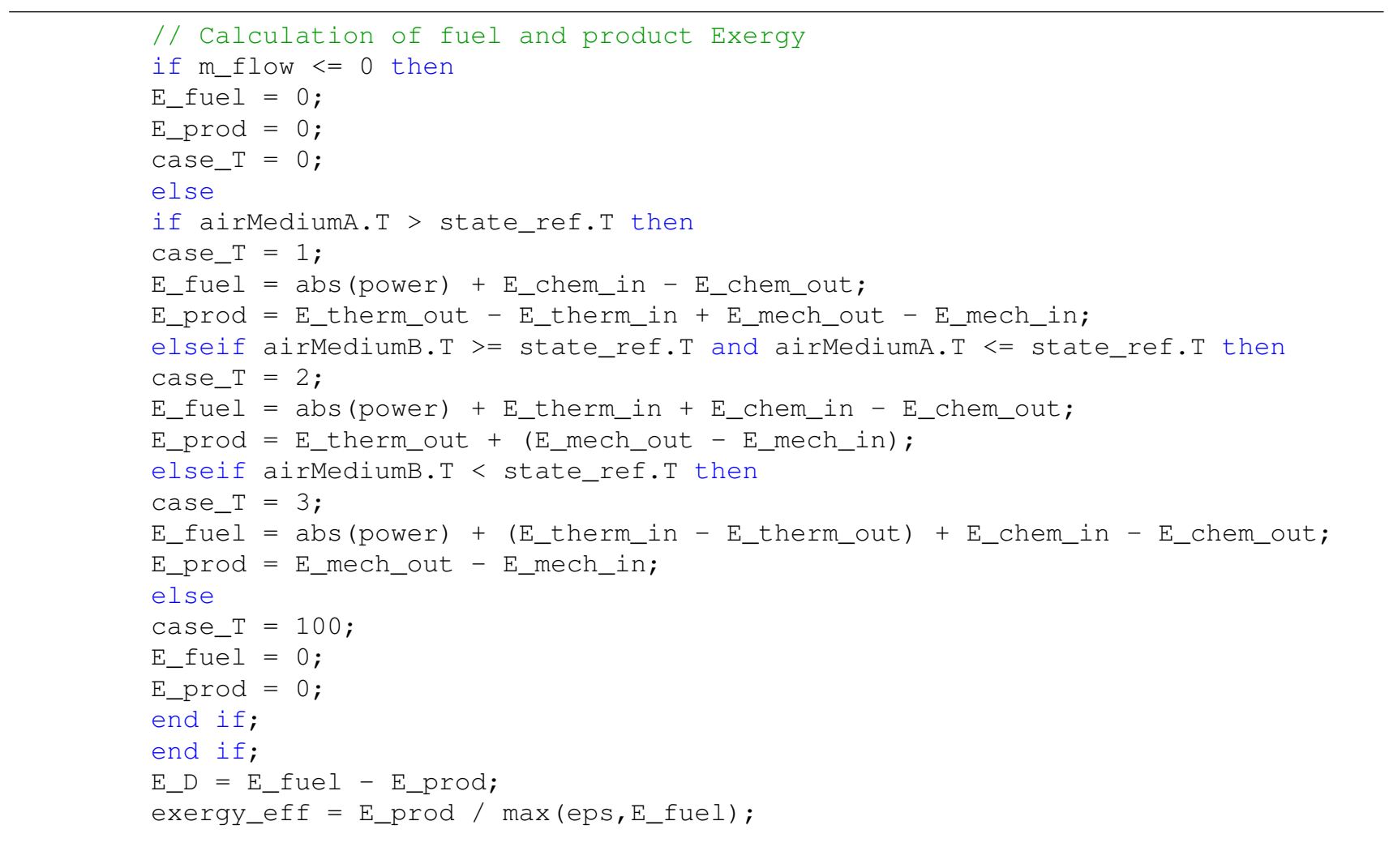

p_ref $=$ p_ref,

X_ref = X_ref);

Besides the thermodynamic state at the connectors, the flow medium, mass flow, reference environment and in this particular case of the compressor, the power is transferred. The propagation of the thermodynamic state enables to be independent from the type of connectors used for the system modeling. The same applies for the flow medium. Here, the same medium model that is used for the component is propagated to the exergy sensor. As long as the medium model provides basic equations similar to the MSL, any medium model can be used. All calculations for the exergy flows and the definitions of the exergy rates depending on the operation condition (Table 1) happen within the sensor model. First the specific exergy at the inlet and outlet of the component are calculated. Function models provide the algorithms to determine the specific exergy split into its thermal, mechanical and chemical parts. Listing 1 shows how the function call works. The thermodynamic states at the connector and of the reference environment are committed to the function and the specific exergy values are returned. Following the exergy flows are calculated depending on the dry air and mass flow. The exergy flows of thermal $\left(\dot{E}^{\mathrm{T}}\right)$, mechanical $\left(\dot{E}^{\mathrm{M}}\right)$, and chemical $\left(\dot{E}^{\mathrm{Ch}}\right)$ parts, and the exergy of work $(\dot{W})$ are then used to formulate the fuel and product balances. The Modelica code of the compressor exergy sen- sor for the exergy rates of Table 1 is presented in Listing 2. It must be mentioned that the thermal and mechanical exergy flows combined are regarded as physical exergy flow: $\dot{E}^{\mathrm{T}}+\dot{E}^{\mathrm{M}}=\dot{E}^{\mathrm{Ph}}$. This approach allows the calculation of the exergy destruction within each component. The user integrates the sensor to the component level and does not have to care about the formulation of the exergy equations. The recent exergy library contains a sensor model for each category listed in Table 2.

\subsection{System level}

A WorldEx model controls the exergy analysis on system level. It is responsible for the definition of the reference environment and collects the exergetic information for the exergy balance on system level. The reference environment can be either defined with fixed values or it can be linked to an environment model with variable conditions.

The exergy balance for the total system is formulated by Equation (3). The exergy flows for the total fuel, product and losses must be defined by the user as they depend on the system architecture. For each of the exergy flows, sensor models are available to integrate them to fluid flows. Similar to mass flow sensors, they must be implemented within the appropriate fluid stream. The exergy of the stream is calculated without any impact on the flow. In some cases it is not possible to use fluid sensors to catch fuel, product or loss exergy of the system, e.g. pure power 
that is supplied to or extracted from the system. For such cases the GUI of the world model provides input boxes for each part of the exergy balance where the variable names can be entered. A mixed usage of sensors and entered variable names is possible as all parts are captures and summed up automatically.

The capability of automatically collecting the exergy data for the system balance requires the identification of all implemented exergy objects. Unfortunately, during runtime, Modelica does not provide such capabilities to screen a system model for its data structure. With the help of the UID Library (Hellerer and Buse, 2017) all exergy sensors are equipped with a unique identifier which can be identified on system level and used for the system balance. The following listing shows the implementation of the unique identifier to the exergy sensor for the implementation to the components:

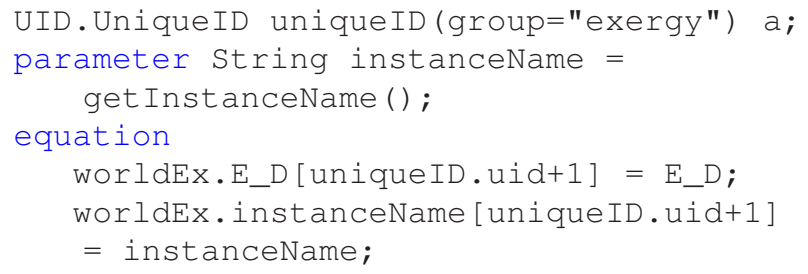

The UniqueID model is added to the sensor model and assigned with a group. A unique integer value uid is then provided within the group. In this way, it is possible to propagate the exergy destruction calculated within each sensor and the instance name of the component to the WorldEx model. The uid value starts at 0 and is in the range $[0 \ldots$ total $[$. The World model contains a GroupTotal object that provides the total number of values assigned within a certain group:

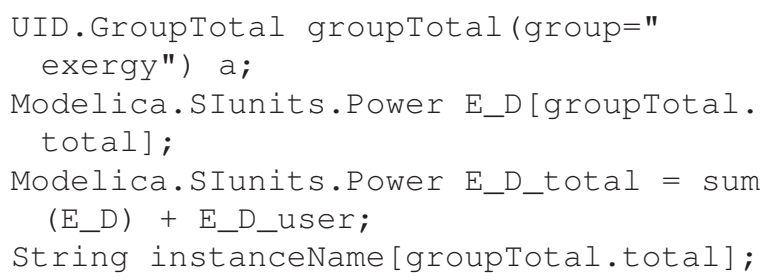

This allows to summarize the single values of the exergy destruction to the total destroyed exergy within the considered system. The collection of the fuel, product and loss exergy flows works in a similar way using additional GroupTotal objects having different group names assigned.

The WorldEx model provides the list of the collected instance names of the components that have an exergy sensor implemented in the Dymola Message Window and writes them into an extra text file.

\subsection{Library structure}

The structure of the library is shown in Figure 4. The WorldEx model is available on the top level. Besides the User's Guide and Examples package there are three packages on the top layer. The Functions package includes all functions that are necessary to calculate the exergy flows

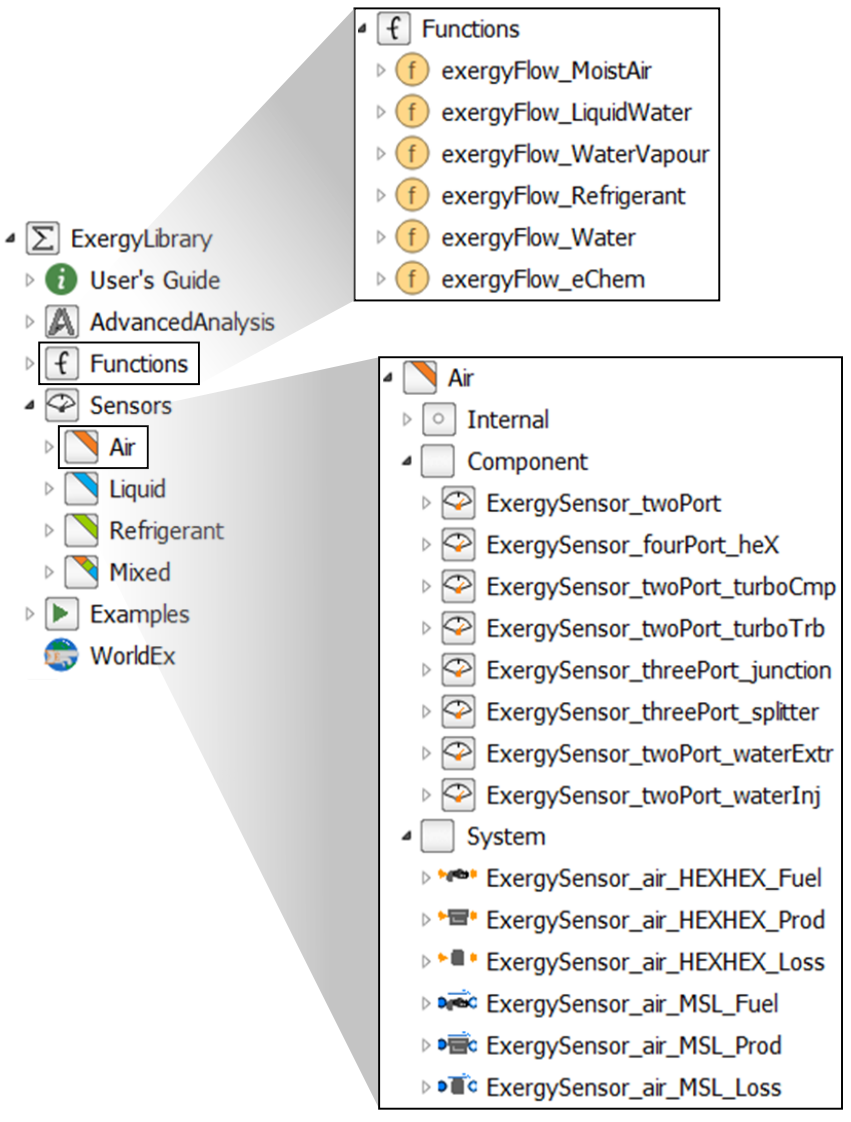

Figure 4. Library structure of the exergy library.

and called from the exergy sensor models (Listing 1). The Sensors package contains all sensor models. It is organized according the fluid medium. Currently there are sensors available for air, liquid, refrigerant, and mixed media. Typical applications for mixed media sensors are for example heat exchangers with two media. All sensors are valid for unidirectional flow. The examples package aims at the understanding of how the sensor models are applied. Finally, there is an additional package for advanced analysis applications that cover aircraft specific calculations such as fuel weight penalties and the calculation of the flow exergy of aviation jet fuel.

The sensor package is further organized in component and system level. The component package contains the sensors for the different component types. Currently eight processes are covered: flow resistances and valves, heat exchangers, power demanding and producing turbo components, junctions such as splitter and mixer, and water extraction and injection. The System package provides sensor models to perform exergy analysis on system level according Equation 3. The sensor models equal mass flow sensors and need to be integrated in between a flow stream. These sensors must be compatible with the infrastructure, i.e. have the same connectors, of the used library. For the moment there are sensors provided that are compatible with the Modelica standard library and a new approach of thermo-fluid modeling that is presented 
in detail by (Zimmer et al., 2018).

\section{Examples}

Within this section, two examples will show how the exergy library is applied to thermo-fluid systems.

\section{Modelica Standard Library}

The simple power generation cycle with moist air as working fluid has already introduced this work in the first section. Cold air is preheated within a heat exchanger and then expanded while passing a turbine to produce power. Figure 1 shows the diagram layer of the Modelica model. On the top layer the WorldEx model and the exergy sensors for the system balance, Loss and Fuel can be seen. The produced power of the turbine states the product of the total exergy balance. The integration of the power value to the exergy balance works by writing the variable name to the GUI of the WorldEx, Figure 5. The exergy sensors

\begin{tabular}{|c|c|c|c|c|c|}
\hline General & Exergy Balance & Add modifers & Attributes & & \\
\hline \multicolumn{2}{|c|}{ E_fuel_user } & & & - $w$ & Fuel exergy for system balance. \\
\hline \multicolumn{2}{|c|}{ E_prod_user } & & HPT.power & - w & Product exergy for system balance. \\
\hline \multicolumn{2}{|c|}{ E_loss_user } & & & - w & Loss exergy for system balance. \\
\hline E_D_us & & & & - $w$ & Additional exergy destruction for sys \\
\hline
\end{tabular}

Figure 5. Extract of the Wor $l d E x$ GUI showing the assignment of the turbine power to the product exergy of the system balance.

for the components are integrated on component level. An example is shown in Figure 3. Once the exergy sensors have been implemented, the model can be simulated in the usual way.

In the beginning of the simulation a text file "ExComponentNames_*modelName*.txt", where *modelName* is replaced with the name of the simulated model, is created. It lists all components of the simulated model with exergy sensors inside (Figure 6) and a list of used exergy sensors for the system balance, i.e. fuel, product and loss. In this example there are four components, the turbine (HPT), the heat exchanger (hx_HP) and two pipes, listed with their names and paths to the exergy sensor and two fuel and loss exergy sensors. The path to the exergy sensors show that the naming of the sensors is the same independent of the exergy sensor model. A default name is set for all exergy sensor models. Taking an example from Figure 4, usually when the "ExergySensor_twoPort" model is dropped into a pipe model, the name of the sensor model would automatically be set to "exergySensor_twoPort". To simplify an optional post processing of the results by using the generated *.mat file, the exergy sensor name was set to "exergySensor" per default for all models. For the exergy analysis the type of exergy sensor is not important any more as the appropriate balances are included already. The power generation cycle model was simulated for $50 \mathrm{~s}$. It starts at steady state condition and then the mass flow of the hot air supply is reduced between 10s and 40s. The plots of the results are shown in Figure 7. The model is
] ExComponentNames_PowerGenerationSystem_ModelicaPaper.tx...

Datei Bearbeiten Format Ansicht ?

Total Number of components containing exergy sensors $=4$ Structure of vectors based on E_D:

[1] HPT

[2] hX_HP

[3] pipe

[4]pipe1

Fuil paths to exergy sensors:

PowerGener at i onsystem_Model 1 i caPaper. HPT. exer gysensor

PowerGener at ionsyst em_ModelicaPaper. hX_HP. exergysensor

PowerGener at ionsystem_Model icapaper. pipe. exergysensor

Power Gener at ionsystem_Modelicapaper. pipe1. exergysensor

Total Number of fuel exergy sensors $=2$

Structure of E_fuel vector:

[1] PowerGener at i onsystem_Modelicapaper.Fue1_hot_in [2] PowerGener at ionsyst em_Modelicapaper. Fuel_cold_in

Total number of prod exergy sensors $=0$

Total Number of prod exergy

Structure of E-prod vector:

structure of E_loss vector:

Structure of E_loss vector:

Iicapaper.Loss_hot_out [2] PowerGener at i onsystem_Model i capaper. Loss_cold_out

Figure 6. Generated text file with list of components equipped with exergy sensor and list of system exergy sensor models for fuel, product and losses.

just an exemplary application and hence the quality of the results is not further discussed here. But it can be captured that the exergetic values on system level are stored and available within the WorldEx model. The results of the fuel and product balances on component level can be found in the variable browser at the paths listed in Figure 6.

\section{Directed thermo-fluid flows using HEXHEX}

In the beginning of this paper the requirement of generic and library independent compatibility was emphasized. To show this capability of the exergy library, the second example is modeled using a new approach for robust modeling of directed thermo-fluid flows. This methodology has recently been developed at our institute (Zimmer et al., 2018) with the aim to provide robust modeling for complex networks such as aircraft environmental control systems. The example architecture is much more complex than the example modeled with the MSL, Figure 1. Here the exergy library shows its real advantage for the analysis of large systems.

Figure 9 shows the diagram layer of the second example, an electric driven vapor cycle pack (eVCP) architecture. The architecture is derived from a patent publication (Golle et al., 2016). Unlike the original architecture, the vapor cycle was simplified. The original vapor cycle has an additional evaporator flown through with recirculated air from the cabin. Unlike conventional bleed air driven air cycle packs (Bender, 2017), unconditioned outside air instead of bleed air from the engine enters the eVCP. The cold and low pressure air is compressed in a first stage before it passes the primary heat exchanger (PHX). A second compressor further raises the pressure and temperature before entering the reheater. The main heat exchanger, mounted in the ram air channel, is passed before the evaporator cools the air. In case the saturation temperature is exceeded, a water separator extracts the condensate and 


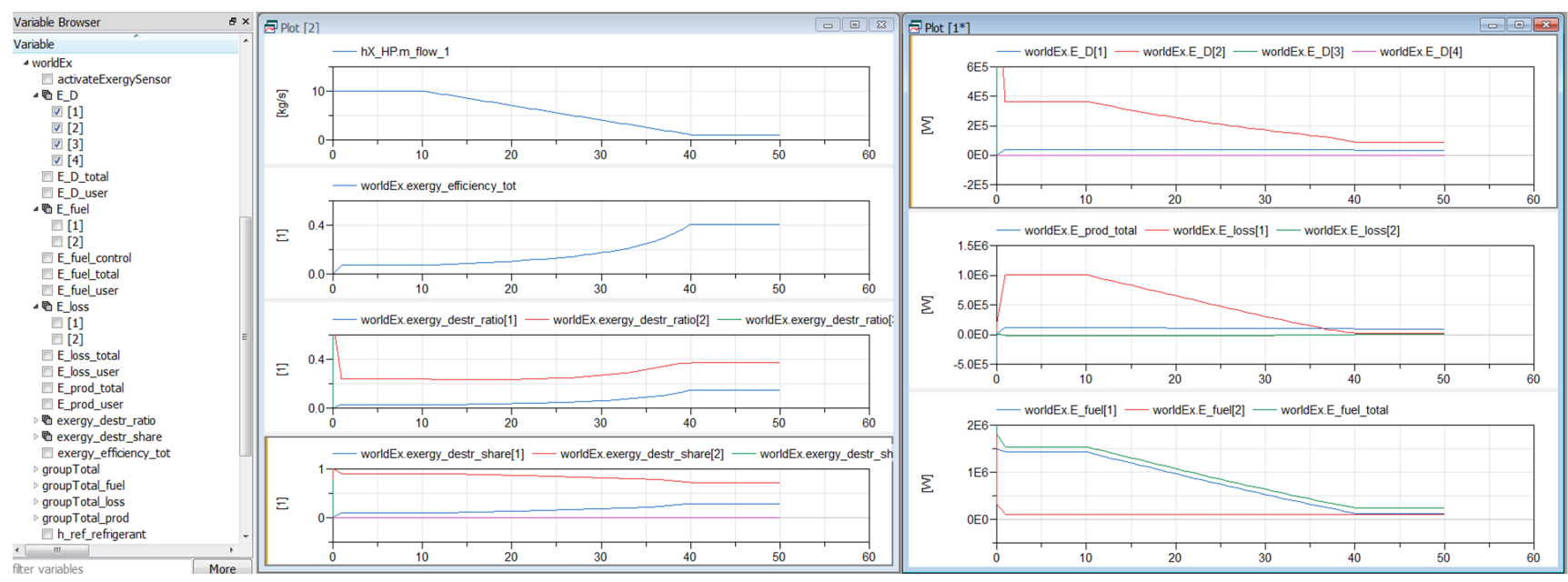

Figure 7. Simulation results of the power generation cycle showing exergy values plotted.

leads it to the water injector located in the the ram air channel upstream the vapor cycle condenser. Before the conditioned fresh air is expanded in the turbine, it is reheated in the reheater. The discharged fresh air meets the recirculated cabin air in the mixing unit downstream the pack. The ram air channel functions as heat sink and is feed with air from the outside. During ground operations, the air flow is provided by a fan located near the ram air outlet. Contrary to a bleed air driven pack which is autonomously driven, all turbo-machines within the eVCP are electrically powered. Within the electric driven vapor cycle pack model, a total number of 30 components with exergy sensors was identified by the exergy library. Figure 8 shows an extract of the text file. Although there are 30 components listed, the exergy sensor was not integrated 30 times manually to the component models. The work has to be done once for each different component class and can then be reused without limitations. The simulation results are available in the variable browser, and stored in the *.mat file, as presented for the MSL example in Figure 7.

\section{Conclusion}

Risen from the idea to do exergy analysis for aircraft environmental control systems within model-based design, a solution had to be found how to integrate exergybased methods into the model-based design environment of Modelica. With this motivation, the presented exergy library was developed. The exergy analysis treats every component different, depending on its aim and particular energy conversion process. The derived requirements for such a library coming from both, the exergetic and modelbased design, parties resulted in the presented work. An exergy sensor model is implemented into the component model and linked to the entering and exiting energy flows. The sensor then does all calculations for the component level exergy analysis and propagates its instance name and exergy destruction to a WorldEx model. Concurrently, the WorldEx model provides the reference environment for

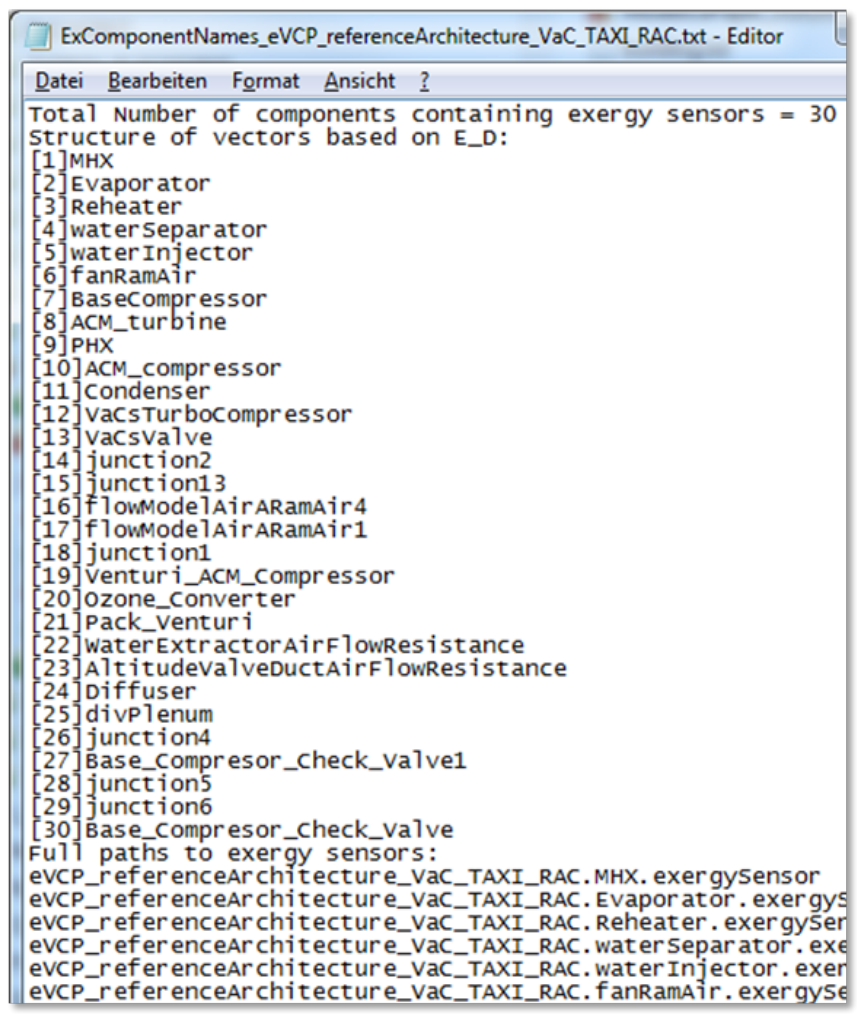

Figure 8. Generated text file of eVCP architecture with list of components equipped with exergy sensor and list of system exergy sensor models for fuel, product and losses.

all exergy sensors. The exergy sensor model can be applied independently from the thermo-fluid modeling approach. This was achieved by linking the sensor model not by connecting any fluid connectors but by propagating the thermodynamic states of the entering and exiting fluid flows. The exergy balance on system level can be performed by using additional exergy sensors that work similar to mass flow sensors and need to be connected within the appropriate fluid stream. Unfortunately, these models have to be compatible with the fluid library, i.e. have 


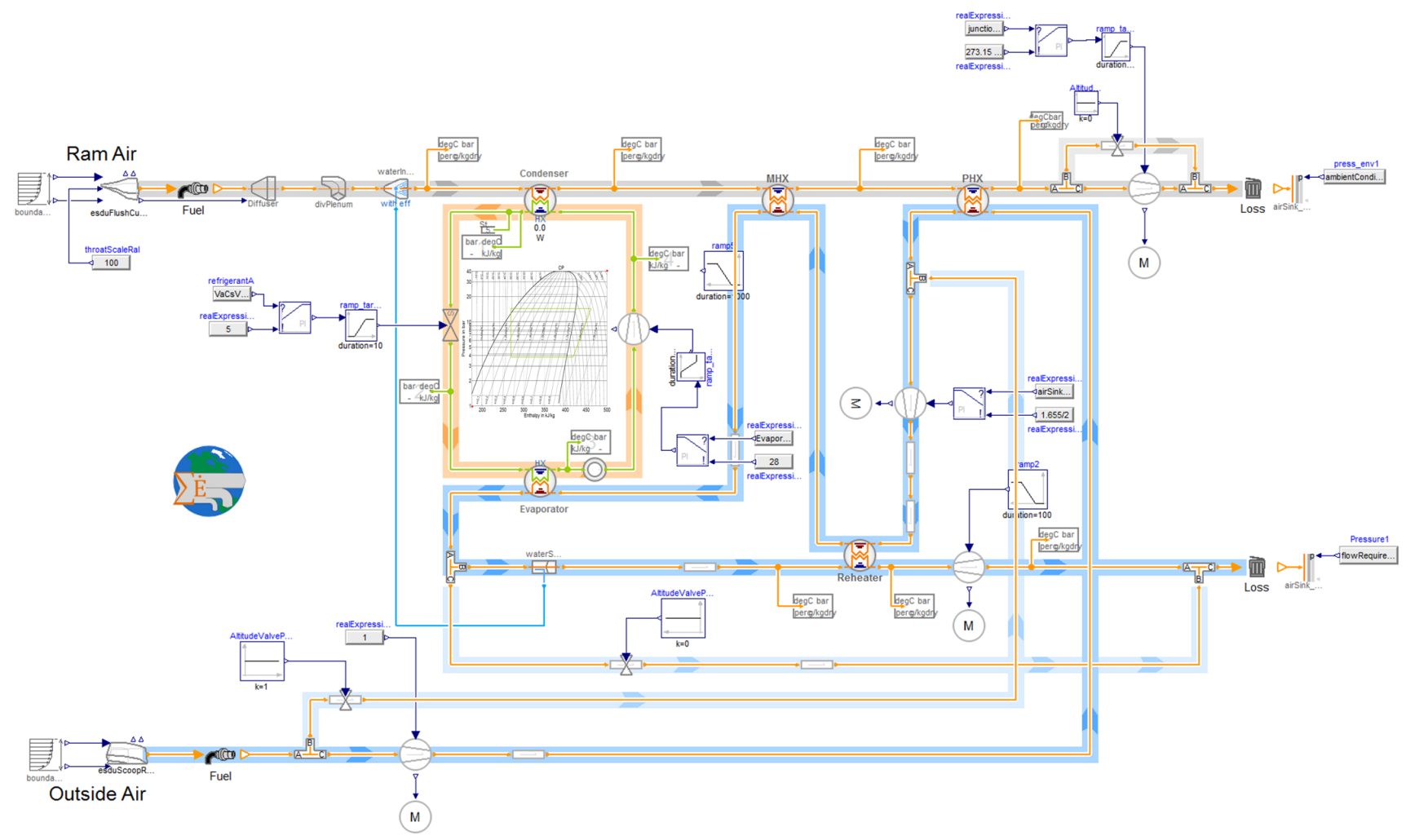

Figure 9. Modelica diagram of electric driven vapor cycle pack architecture modeled using the HEXHEX library.

the same or compatible connectors. The current version of the exergy library provides sensors that can be used with the Modelica Standard Library and the approach of HEXHEX (Zimmer et al., 2018). Not accessible energy streams that should be included to the system balance can be added by writing their variable names into the GUI of the WorldEx model. Here a box is provided for exergy destruction, fuel, product and loss exergy. Additional evaluation numbers such as exergy destruction rate (component and system level) or the ratio of $E_{\mathrm{D}, k} / E_{\mathrm{D}, t o t}$ are provided by the WorldEx model.

With the experience gained during the development of this exergy library, one can say that there is no universal solution as exergy-based methods by their nature, have the individual aspect on the component's aim. But there usually is a limited number of energy conversion processes and components classes. The work for integrating the exergy sensors to the components, needs to be done once and one can benefit from it any time after. The structure of the library allows an easy extension for new energy conversion processes. The advantage of this concept is that the exergy analysis is totally detached from the component development and behavior and allows an as individual use as possible for the modeler.

\section{Remarks}

The current version of the exergy library uses mainly the media models of the Modelica Standard Library. For moist air, water and single gases that can be treated as ideal gases, the provided models of the MSL might be suf- ficient. The second example architecture presented here, has a vapor cycle included that runs with refrigerant. An additional media library was used. The linchpin of the exergy analysis is the correct calculation of the thermodynamic properties. The current thermodynamic state record of the MSL or most other medium models does supply only basic properties which are not sufficient for an exergy analysis (i.e. specific entropy). Therefore, an extended thermodynamic state record was created in the exergy library by using the appropriate equations from the medium models. To further ensure a generic applicability of the exergy library for different kinds of fluids, it is recommended to provide standardized formulation and naming of equations for the thermodynamic properties. Unfortunately, this is not the case for all medium models.

\section{References}

Ansys. Ansys, 2018. URL https://www. ansys.com/.

aspentech. Aspen plus, 2018. URL https://www . aspentech.com/products/engineering/ aspen-plus.

Adrian Bejan, George Tsatsaronis, and Michael J. Moran. Thermal design and optimization. A Wiley-Interscience publication. Wiley, New York, 1996. ISBN 978-0471-58467-4. URL http://www.1oc.gov/catdir/ description/wiley032/95012071.html.

Daniel Bender. Desa - optimization of variable structure modelica models using custom annotations. In Francesco Casella and Dirk Zimmer, editors, Proceedings of the 7th International Workshop on Equation-Based Object-Oriented Mod- 
eling Languages and Tools - EOOLT '16, pages 45-54, New York, New York, USA, 2016. ACM Press. ISBN 9781450342025. doi:10.1145/2904081.2904088.

Daniel Bender. Integration of exergy analysis into modelbased design and evaluation of aircraft environmental control systems. Energy, 137:739-751, 2017. ISSN 0360-5442. doi:10.1016/j.energy.2017.05.182.

Hilding Elmqvist, Hubertus Tummescheit, and Martin Otter. Object-oriented modeling of thermo-fluid systems. In The Modelica Association, editor, Proceedings of the 3rd International Modelica Conference, pages 269-286, 2003.

F-Chart. Ees, 2018. URL http://www. fchart.com/ ees/.

Stefan Golle, Ullrich Hesse, Enrico Klausner, Frank Klimpel, Hans Brunswig, and Mario Raddatz. Betriebsphasenabhängig steuerbare flugzeugklimaanlage und verfahren zum betreiben einer derartigen flugzeugklimaanlage, 2016.

P. Grassmann. Zur allgemeinen definition des wirkungsgrades. Chemie Ingenieur Technik - CIT, 22(4):77-80, 1950. doi:10.1002/cite.330220402.

Matthias Hellerer and Fabian Buse. Compile-time dynamic and recursive data structures in modelica. In Dirk Zimmer and Bernhard Bachmann, editors, Proceedings of the 8th International Workshop on Equation-Based Object-Oriented Modeling Languages and Tools - EOOLT '17, pages 8186, New York, New York, USA, 2017. ACM Press. ISBN 9781450363730. doi:10.1145/3158191.3158205.

Andrea Lazzaretto and George Tsatsaronis. Speco: A systematic and general methodology for calculating efficiencies and costs in thermal systems. Energy, 31(8-9):1257-1289, 2006. ISSN 0360-5442. doi:10.1016/j.energy.2005.03.011.

Dirk Limperich, Marco Braun, Kathrin Prölß, and Gerhard Schmitz. System simulation of automative refrigeration cycles. Proceedings of the 4th International Modelica Conference, 2005.

Andreas Pfeiffer. Optimization library for interactive multicriteria optimization tasks. In: Proceedings of the 9th International Modelica Conference, 2012.

Roozbeh Sanghi, Jahangiri Pooyan, Alexander Thamm, Rita Streblow, and Dirk Müller. Dynamic exergy analysis - part i: Modelica-based tool development. Building Simulation and Optimization Conference, 2014.

Michael Sielemann, Tim Giese, Bettina Oehler, and Martin Otter. A flexible toolkit for the design of environmental control system architectures. In: Proceedings of the First CEAS European Air and Space Conference, 2007. Berlin.

The Modelica Association. The modelica language specification: Version 3.3, 2013. URL https: / / www.modelica.org/documents / Modelicaspec33Revision1.pdf.

G. Tsatsaronis and T. Morosuk. Exergy-based methods applied to the chain natural gas - lng - natural gas. Proceedings of the 3rd International Exergy, Life Cycle Assessment, and Sustainability Workshop \& Symposium (ELCAS3), July 2013.
George Tsatsaronis. Definitions and nomenclature in exergy analysis and exergoeconomics. Energy, 32(4):249-253, 2007. ISSN 0360-5442. doi:10.1016/j.energy.2006.07.002.

Michael Wetter, Wangda Zuo, Thierry S. Nouidui, and Xiufeng Pang. Modelica buildings library. Journal of Building Performance Simulation, 7(4):253-270, 2014. ISSN 1940-1493. doi:10.1080/19401493.2013.765506.

Stefan Wischhusen, Bruno Lüdemann, and Gerhard Schmitz. Economical analysis of complex heating and cooling systems with the simulation tool hksim. In: Proceedings of the 3rd International Modelica Conference, 2003.

Dirk Zimmer and Daniel Schlabe. Implementation of a modelica library for energy management based on economic models. In The Modelica Association, editor, Proceedings of the 9th International Modelica Conference, pages 133-142, 2012. doi:10.3384/ecp12076133.

Dirk Zimmer, Daniel Bender, and Alexander Pollok. Robust modeling of directed thermofluid flows in complex networks. In: Proceedings of the 2nd Japanese Modelica Conferences, 2018. 